\title{
Stem cell niche as a prognostic factor in leukemia
}

\author{
Ga-Young Lee, Jin-A Kim \& Il-Hoan $\mathrm{Oh}^{*}$ \\ The Catholic High-Performance Cell Therapy Center \& Department of Medical Lifescience, College of Medicine, The Catholic University of \\ Korea, Seoul 06591, Korea
}

\begin{abstract}
Despite high interests on microenvironmental regulation of leukemic cells, little is known for bone marrow (BM) niche in leukemia patients. Our recent study on BMs of acute myeloid leukemia (AML) patients showed that the mesenchymal stromal cells (MSCs) are altered during leukemic conditions in a clinical course-dependent manner. Leukemic blasts caused reprogramming of transcriptomes in MSCs and remodeling of niche cross-talk, selectively suppressing normal primitive hematopoietic cells while supporting leukemogenesis and chemo-resistance. Notably, differences in BM stromal remodeling were correlated to heterogeneity in subsequent clinical courses of AML, i.e., low numbers of mesenchymal progenitors at initial diagnosis were correlated to complete remission for 5-8 years, and high contents of mesenchymal progenitor or MSCs correlated to early or late relapse, respectively. Thus, stromal remodeling by leukemic cell is an intrinsic part of leukemogenesis that can contribute to the clonal dominance of leukemic cells over normal hematopoietic cells, and can serve as a biomarker for prediction of prognosis. [BMB Reports 2015; 48(8): 427-428]
\end{abstract}

Accumulating studies have pointed out the importance of bone marrow microenvironment in regulating the behavior and cell fate of normal and malignant hematopoietic cells. The microenvironmental regulation of normal hematopoietic stem cells (HSCs) mostly occurs in a specialized architecture of bone marrows (BM) referred to as stem cell niche, where the majority of HSCs reside and interact with stromal cells. Studies have shown that two types of niche exist in the bone marrow:

*Corresponding author. E-mail: iho@catholic.ac.kr

http://dx.doi.org/10.5483/BMBRep.2015.48.8.152

\section{Received 19 July 2015}

Keywords: Niche, Leukemia, Prognostic factor, Biomarker

Abbreviations: HSC, hematopoietic stem cells; BM, bone marrow; LSC, leukemia stem cells; AML, acute myeloid leukemia; CXCL-12, C-X-C motif ligand 12

Perspective to: Kim et al (2015), Microenvironmental remodeling as a parameter and prognostic factor of heterogeneous leukemogenesis in acute myelogenous leukemia, Cancer Research, 75 (11) 2222-2231. Doi: 10.1158/0008-5472.CAN-14-3379). endosteal osteoblastic niche and vascular/peri-sinusoidal niche. Although these two niches are geographically distinct, they share common cellular compositions. They are mostly comprised of mesenchymal stromal cells with participation of sinus endothelial cells and other cellular components. These niche cells are engaged in cross-talks with normal HSCs primarily through CXCL-12/CXCR-4, Jagged-1/Notch, and other signaling pathways (reviewed by Oh \& Kwon, Stem Cells 2010, 28 (7); 1243-9, doi: 10.1002/stem.453). HSCs are regulated for self-renewal, quiescence, survival, and differentiation in the context of microenvironmental regulation.

Acute myeloid leukemia (AML), a heterogeneous clonal disorder of hematopoietic progenitor cells, can lead to excessive proliferation of malignant progenitor cells. Recent studies have shown that leukemia stem cells (LSCs), a subpopulation of leukemia cells, are responsible for the development, resistance, and relapse of leukemic cells in patients. LSCs arise from the transformation of HSCs or re-acquisition of self-renewing potential in committed progenitors. They share some common properties of normal HSCs. When transplanted into mice, these LSCs can engraft in bone marrow and compete for niche with normal HSCs, suggesting that these LSCs might similarly interact with niche cells in bone marrow.Thus, emerging concept on LSC has pointed to the influence of stem cell niche for leukemia cells. However, key issues remain unclear is the impact of leukemic conditions on the niche and the significance of BM niche on leukemogenic process itself. Regarding the niche under leukemic conditions, several lines of animal studies have shown that leukemia cells can alter the condition of mesenchymal niche. A transgenic model of chronic myeloid leukemia has exhibited defective homing and retention of HSCs in the niche due to decreased CXCL12 in BM MSCs. Similarly, MSCs or osteoblastic cells are altered in the bone marrow of leukemia animals induced by bcr-abl. These studies have shown that the mesenchymal niche is altered under leukemogenic conditions. However, despite these observations, the clinical relevance of niche remodeling under leukemic conditions have been unclear until a systemic analysis on human AML patients' bone marrows by Kim et al. (Kim et al. Cancer Research, 2015, 75 (11) 2222-2231. Doi: 10.1158/0008-5472. CAN-14-3379). They revealed that human BMs of AML patients indeed exhibited a cellular shift in mesenchymal niche characterized by the loss of mesenchymal stromal cell (MSC) proliferation and loss of mesenchymal progenitors (CD146+, 


\section{Reprogramming of BM niche by LSC}

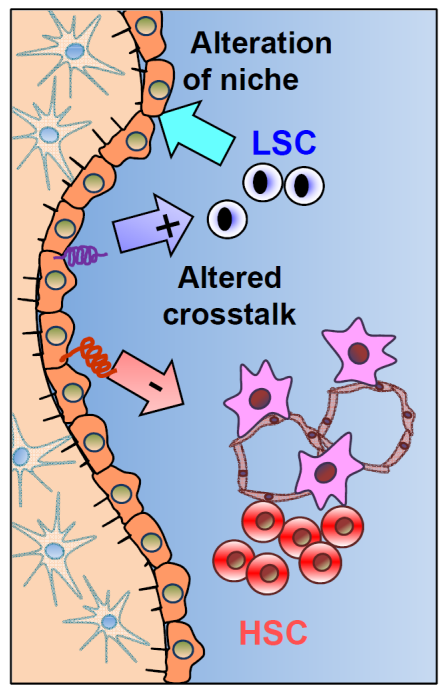

Altered Leukemic Microenvironment

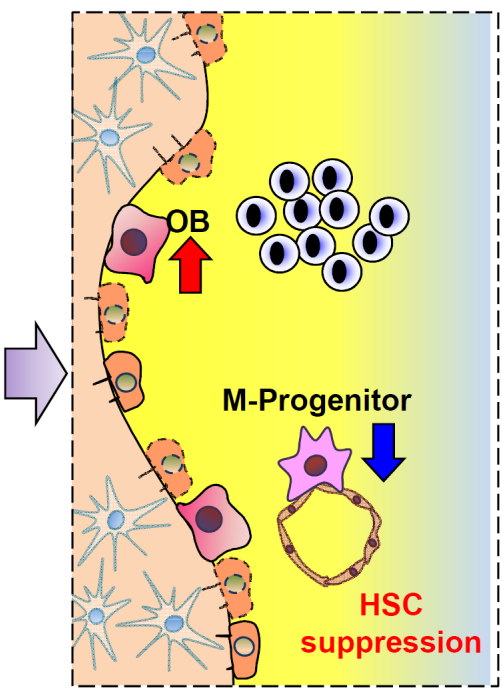

\section{Stromal heterogeneity At Initial diagnosis}

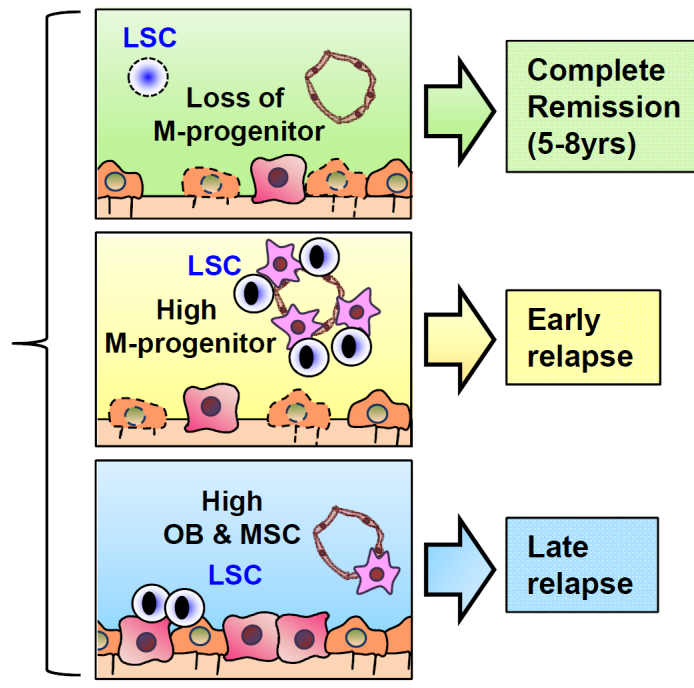

Fig. 1. Schematic illustration of leukemia-induced alterations of niches and their clinical significance. LSC clones developed in bone marrow can induce transcriptional reprogramming of mesenchymal cells and the remodeling of the niche towards a loss of $\mathrm{M}$-progenitors and maturation into osteoblastic cells. Altered niche can provide a distinctive cross-talk to normal HSCs and LSCs in a manner that can selectively suppress normal HSCs $(-)$ but maintain LSCs $(+)$, leading to the dominance of leukemia cells over normal hematopoietic cells. The difference in stromal remodeling at the initial diagnosis is associated with heterogeneity in clinical prognosis after the treatment. Thus, stromal remodeling as a parameter that can influence leukemogenic activity and subsequent clinical course can serve as a prognostic marker in AML.

primitive subsets of MSCs) along with extensive transcriptional reprogramming in MSCs.

Of note, during the reprogramming of mesenchymal niche, the expression of cross-talk molecules (Jagged-1 or CXCL-12) in niche cell was reset in a manner that it can distinctively regulate normal and malignant hematopoietic progenitors to selectively suppress normal HSCs while sparing the leukemogenic activity of leukemia cells. Thus, the mesenchymal niche altered by leukemic cells can contribute to clonal dominance of leukemia cells over normal hematopoietic pool, leading to the loss of normal hematopoietic function, a major cause of mortality under leukemic conditions. Thus, leukemic niche established by leukemic cells should be an intrinsic process of leukemogenesis that can self-reinforce the leukemic process.

Since the leukemic niche exerts a regulatory impact on normal and leukemic cells, one interesting possibility is that it could be a factor that influences the heterogeneity in the clinical course of leukemia patients. Strikingly, our recent study (Kim et al, ibid) has revealed that the difference in the stromal pattern in AML patients at the time of initial diagnosis is associated with different clinical courses for subsequent 5-8 years after the initial treatment (illustrated in Fig. 1). Most BMs in AML patients who maintained complete remission after the ini- tial treatment showed low levels of mesenchymal components. However, BMs of those patients who experienced early $(<1$ year) or late $(>1$ year) relapse exhibited higher levels of mesenchymal progenitors or MSCs, respectively. Thus, BM findings at initial diagnosis can serve as a biomarker to predict the clinical course of AML to identify high-risk patients. Since the identification of risk for relapse has been of major interest for the management of leukemia patients, various markers have been developed for unfavorable prognosis, including cytogenetic abnormality and gene expression pattern of leukemic blasts. Thus, predicting relapse by stromal pattern could provide new approaches for therapeutic planning based on individual microenvironment.

Collectively, microenvironment-based approaches to leukemia should open a horizon for personalized and biology-based treatments towards more efficient leukemia treatment by targeting specific niche conditions in each individual leukemogenic process.

\section{ACKNOWLEDGEMENTS}

This study was supported by NRF, Korea and funded by the Ministry of Science, ICT \& Future Planning (No. 2011-0019352). 